\title{
Journal of Counseling Psychology Principal Reviewers, Contributing Reviewers and Pre-Doctoral Co-Reviewers (January 2017 to September 2018)
}

\section{PRINCIPAL REVIEWERS}

The Editor and Associate Editors wish to thank the following persons who served as principal reviewers for Journal of Counseling Psychology by reviewing a minimum of four manuscripts.

Mary Z. Anderson

Consuelo Arbona

Gary Burlingame

Marilyn A. Cornish

Ryan D. Duffy

Stefanía Ægisdóttir Adriana Aldana

Alexis V. Arczynski

Julie Arseneau

David Atkins

Scott A. Baldwin

Kira Banks

Eran Bar-Kalifa

Sharon Beiman

Lynette H. Bikos

Shachaf Bitan

James F. Boswell

Richard Bränström

Walter Buboltz

Stephanie L. Budge

Jonathan E. Butner

Esteban Cardemil

Grace I. L. Caskie

Shannon Chavez-Korell

Na-Yeun Choi

Ayse Çiftçi

Brian Cole

Collie W. Conoley

Jennifer Cook

Alexandra F. Corning

Eric D. Deemer

Edward A. Delgado-Romero

Sarah S. Dermody

Frank R. Dillon

Robert Elliott

David M. Erekson
Patricia A. Frazier
Kirsten Gonzalez
Bong Joo Hwang
LaRae M. Jome

David M. Erekson
Chetan A. Joshi

Cindy Lee Juntunen

Daniel G. Lannin

$\mathrm{Xu} \mathrm{Li}$

Yun $\mathrm{Lu}$

Ryon C. McDermott

Uma Chandrika Millner
Stephen M. Quintana

Mike Schaub

Eric Swank

Nathaniel G. Wade

Laurel B. Watson

Hui $\mathrm{Xu}$

\section{CONTRIBUTING REVIEWERS}

James M. Fauth

Lisa Y. Flores

Ze'ev Frankel

Myrna L. Friedlander

Kristin Gainey

M. Paz Galupo

Garren Gaut

Omar Carlo Gioacchino Gelo

Simon B. Goldberg

Juan Martin Gomez Penedo

Stephanie Graham

Marie S. Hammond

Ashley Hampton

Nadia T. Hasan

Laurie Heatherington

Melanie Hetzel-Riggin

Clara E. Hill

Mark J. Hilsenroth

Michiyo Hirai

Wai Kai Hou

Carla D. Hunter

Margo A. Jackson

Jack Keefe

Maureen E. Kenny

Brian TaeHyuk Keum

Brian Keum

Eunha Kim

Paul Youngbin Kim

Avi Kluger
Justin Lavner

Mark M. Leach

Ronald F. Levant

James W. Lichtenberg

William Ming Liu

Lorenzo Lorenzo-Luaces

Marvin Lynn

Brent Mallinckrodt

Rayna Markin

Kevin Scott McCarthy

Takuya Minami

Nancy L. Murdock

Dana Nelson

Aviv Nof

Seini O'Connor

Eleanor Pardess

David Pincus

Jae Puckett

Fabian Ramseyer

Luke Rapa

Robert J. Reese

Clarissa Richardson

Brandon Robinson

Julian Rubel

Donna E. Schultheiss

Eleanor K. Seaton

Jocelyn Shu

Andy Siddaway
Stephanie Simon-Dack

Christopher Slaten

Nathan Grant Smith

Nili Solomonov

Joel Steele

Rong $\mathrm{Su}$

Martin Swanbrow Becker

Dawn M. Szymanski

Christina Thai

Idia Thurston

Terence J. G. Tracey

Sherri L. Turner

Tracy L. Tylka

Pål Gunnar Ulvenes

Ellen Vaughan

Chiachih D. C. Wang

Russell Thomas Warne

C. Edward Watkins, Jr.

Serena Wee

Ingrid K. Weigold

Kerrie Wilkins-Yel

Stacey Williams

Susan S. Woodhouse

Kamilah Woodson

Christine J. Yeh

Hyung Chol Yoo

Eunju Yoon

Richard A. Young

\section{CONTRIBUTING PRE-DOCTORAL CO-REVIEWERS}

\begin{abstract}
Alexandra Agiliga
Lydia Ahn

Bonnie Andrews
\end{abstract}

Haley Bell

Aaron Bethea

Jennifer Boike-Armerding

Rachel E. Brenner
Derek Caperton

Rachel Chickerella

Eddie Siu Kwan Chong
Kuo Deng

Keri Frantell 
Abigail Gabriel

Brittany Griffin

Halleh Hashtpari

Patrick Heath

Stefan Jadaszewski

Ling Jin

Sarah Job

Jessicah Johnson

Tim Kaehr
Dakota Kaiser

Colleen Kase

Patty Kuo

Christina Lee

Liat Leibovich

Joanh Li

Ilana Lipsitz

Meredith Maroney

Keiko McCullough

Liza Meredith
Anahvia Moody

Katherine Morales

Zenobia Morrill

Néstor Noyola

Riley Palmer

Laura Purdham

Katheryn Roberson

Tina Soma
Joanna Sullivan

Rebecca Troeger

Carlos Vidales

Shu-Yi Wang

Stephanie Yee

Nelson Zounlome 